\title{
SAÚDE E DESENVOLVIMENTO DA CRIANCCA: A FAMÍLIA, OS FATORES DE RISCO E AS AÇÕES NA ATENÇÃO BÁSICA
}

\section{Health and the child's development: the family, the risk factors and health care program}

\author{
Juliana S. de O. Molinari ${ }^{1}$ \\ Maria de Fátima M. C. Silva ${ }^{1}$ \\ Maria Aparecida Crepaldi ${ }^{2}$
}

\section{Resumo}

O presente estudo tem por objetivo discutir as interseções entre as temáticas da saúde e do desenvolvimento da criança, considerando a família como contexto de desenvolvimento, assinalando a importância da compreensão da influência de diversos sistemas nesse contexto, com base no modelo ecológico de Desenvolvimento Humano apresentado por Urie Bronfenbrenner. Apresentam-se também noções gerais sobre os fatores de risco para o desenvolvimento da criança, finalizando com reflexões sobre as possibilidades de ação na área da saúde, em atenção básica e vigilância do desenvolvimento.

Palavras-chave: Saúde e desenvolvimento da criança, fatores de risco, atenção básica.

\section{Abstract}

The present study aimes to discuss the thematic of health and the child's development, considering the family as development context, marking the importance of the understanding of the influence of several systems in that context, based on the ecological model of Human Development presented by Urie Bronfenbrenner. General notion about the risk factors and protection for the child's development are presented, concluding with reflections about the action possibilities in the health area, emphasizing the surveillance of the child's development.

Keywords: Health and the child's development, risk factors, health care program.

\footnotetext{
Alunas especiais do Programa de Pós-Graduação em Psicologia. Universidade Federal de Santa Catarina.

2 Dra. em Psicologia. Docente do Programa de Pós-Graduação em Psicologia. Universidade Federal de Santa Catarina. Endereço para contato: Universidade Federal de Santa Catarina Campus Universitário - Trindade - Centro de Filosofia e Ciências Humanas - Departamento de Psicologia. Florianópolis - SC - CEP: 88040-970 .

E-mail: crepaldi@cfh.ufsc.br
} 


\section{Introdução}

O crescimento das desigualdades sociais tem favorecido a ampliação das dificuldades e conflitos vividos pelo contexto familiar e, dessa forma, vem colocando cada vez mais em risco o desenvolvimento saudável das crianças e adolescentes em nossa sociedade.

Por outro lado, o atual desenvolvimento dos sistemas de saúde tem buscado implementar ações que atendam a tais dificuldades. A partir da década de 80 , cresce no Brasil uma reflexão dos órgãos oficiais de assistência à infância visando à preservação da saúde da criança. As políticas públicas no setor de saúde têm se destacado pela efetiva proposta de mudanças, no entanto, a consolidação desse processo depende da crescente cooperação entre governo e sociedade. Sob essa ótica surge, em 1991, o Programa de Saúde da Família (PSF), que faz da família principal foco de atenção, considerando-a a partir do contexto a que pertence (Brasil. Ministério da Saúde, 1997).

Quando pensamos na saúde da criança, além de incluir a família, cabe refletir sobre o desenvolvimento infantil em sua integralidade e entendendo-o como um processo multifacetado, além dos indicadores de doença e mortalidade. Dessa forma, não estamos considerando a saúde da criança como a simples ausência de doença, mas como a vivência de um processo de desenvolvimento adequado em diversos aspectos.

Da mesma maneira, quando pensamos em atenção básica e ações de promoção à saúde, é preciso ir além das ações específicas baseadas nos indicadores de morbi-mortalidade, visando a promover o desenvolvimento saudável em vários aspectos e de forma mais ampla, considerando a saúde física e mental de forma integrada.

O objetivo deste trabalho é discutir as interseções entre as temáticas da saúde e desenvolvimento da criança, no contexto das ações de atenção básica em saúde, considerando a família como contexto privilegiado de desenvolvimento e enfocando, além de aspectos físicos, também os aspectos do desenvolvimento mental, psicológico e social da criança de forma integrada. Para embasar a discussão, apresentaremos noções gerais sobre a teoria ecológica de desenvolvimento humano e sobre fatores de risco e de proteção para o desenvolvimento infantil, procurando trazer contribuições de pesquisas e trabalhos atuais realizados nessa área e refletir sobre as possibilidades de ações na atenção básica.

\section{Desenvolvimento infantil e o modelo ecológico}

É praticamente consenso entre os pesquisadores, estudiosos e profissionais da área que o desenvolvimento infantil é um processo complexo e multideterminado. No conceito apresentado por Miranda, Resegue e Figueiras (2003), desenvolvimento infantil consiste em um processo dinâmico que se inicia na vida intra-uterina e envolve vários aspectos: o crescimento físico, a maturação neurológica e a construção de habilidades relacionadas ao comportamento e às esferas cognitiva, social e afetiva da criança. Visa à competência para responder às suas necessidades e às do seu meio.

Considerando os múltiplos fatores envolvidos e a complexidade do processo de desenvolvimento humano, adotamos a perspectiva ecológica ou ecossistêmica na tentativa de melhor compreendê-lo e abordá-lo neste trabalho. Esta perspectiva considera os diversos sistemas interdependentes (família, grupos, comunidade e sociedade em geral) que influenciam o desenvolvimento da criança, procurando compreender as interações entre a criança e o ambiente em seus vários contextos.

O conceito de ambiente, segundo Zamberlam e Biasoli-Alves (1996, p. 27)

...envolve a dinâmica das relações no que tan-
ge a eventos físicos ou sociais, ou a combina-
ção deles. Propriedades relativas a vários as-
pectos do ambiente, tais como: complexidade,
inovação, interrupção de expectativas não são
determinadas pelos componentes do meio físi-
co, em si, mas resultantes de diversas formas de
interação com a criança que age nesse meio e
que é, também, um organismo ativo, capaz de
captar e ajustar informações desse ambiente no
sentido de alterá-las ou mantê-las.

Urie Bronfenbrenner é um dos principais autores que postula a visão ecossistêmica e destaca-se em seu ponto de vista a idéia de uma interdependência de processos múltiplos na compreensão do desenvolvimento humano. O modelo ecológico proposto por Bronfenbrenner traz a representação de uma inter-relação entre subestruturas sistêmicas caracterizando vários níveis de influência sobre a pessoa em desenvolvimento (Zamberlam \& Biasoli-Alves, 1996). 
O termo ecologia (palavra grega - oikos) significa moradia, habitação, abrangendo as relações dos organismos com o meio físico e não físico. Poderíamos dizer que é a ciência que trata das relações entre os seres vivos e o ambiente em que vivem. (Franco \& Bastos, 2002; Zamberlam \& Biasoli-Alves, 1996).

Zamberlam e Biasoli-Alves (1996, p.111) destacam que: "A Psicologia Ecológica é vista na atualidade como um campo interdisciplinar amplo, que enfoca as relações homem-ambiente do ponto de vista de diversas áreas como, a da Sociologia Urbana, Geografia Humana, Arquitetura e Planejamento Ambiental..." No entanto, há uma distinção, uma vez que a psicologia ecológica enfatiza o estudo de processos psicológicos básicos, evidenciando a interdisciplinaridade para a compreensão das relações.

Segundo Bronfenbrenner (1996, p. 18), entender o desenvolvimento humano "exige mais do que a observação direta do comportamento..., requer um exame de sistemas de interação múltiplas entre pessoas, não limitado a um único ambiente, deve levar em conta aspectos além da situação imediata que contém o sujeito". O autor se refere a essa perspectiva científica evolutiva como "a ecologia do desenvolvimento humano".

A ecologia do desenvolvimento humano envolve o estudo científico da acomodação progressiva, mútua, entre um ser humano ativo, em desenvolvimento, e as propriedades mutantes dos ambientes imediatos em que a pessoa em desenvolvimento vive, conforme esse processo é afetado pelas relações entre esses ambientes, e pelos contextos mais amplos em que os ambientes estão inseridos (Bronfenbrenner, 1996, p. 18).

O modelo bio-ecológico de Bronfenbrenner (1996), também chamado de ecossistêmico, sugere a inter-relação entre subsistemas socialmente organizados que ajudam a apoiar e orientar o desenvolvimento humano.

Antes de caracterizar os subsistemas, cabe apresentar um conceito básico do modelo apresentado por Bronfenbrenner, que é o de processos proximais. Estes se referem aos processos de interação recíproca progressivamente mais complexas entre um organismo humano ativo e as pessoas, objetos e símbolos em seu ambiente imediato. Ou seja, este autor afirma que o desenvolvimento acon- tece por processos de interação entre a criança e todos os níveis de influência do meio ambiente (Halpern \& Figueiras, 2004).

A noção de sistema ecológico composto por cinco subsistemas pode ser visualizada como um local de estruturas aninhadas, uma dentro da outra, sendo elas do nível mais profundo ao mais externo (Bronfenbrenner, 1994, 1996):

- Microssistema: é um padrão de atividades, papéis sociais e relações interpessoais experimentadas pela pessoa em desenvolvimento em um dado ambiente. Exemplos de tais ambientes seriam a família, a escola, o grupo de iguais e o local de trabalho.

- Mesossistema: compreende as relações que ocorrem entre dois ou mais ambientes contendo a pessoa em desenvolvimento, como, por exemplo, as relações entre a casa e a escola, a escola e o local de trabalho, etc. Um mesossistema é um sistema de microssistemas.

- Exossistema: compreende as ligações entre dois ou mais ambientes, sendo que pelo menos um deles não contém a pessoa em desenvolvimento, mas no qual ocorrem eventos que a influenciam indiretamente. Um exemplo seria, considerando uma criança, a relação entre a casa e o local de trabalho do pai ou da mãe.

- Macrossitema: padrão que abrange micro, meso e exossistemas característicos de uma dada cultura, com referência aos sistemas de crenças, conjuntos de conhecimentos, recursos materiais, costumes, estilos de vida, etc.

- Cronossistema: abrange mudanças em relação ao tempo não apenas nas características das pessoas, como também no ambiente em que esta vive, como por exemplo, mudanças no curso de vida e estrutura da família.

Considerando amplitude e complexidade do modelo apresentado, fica mais evidente a necessidade de considerar a importante influência de cada sistema no desenvolvimento do indivíduo e percebe-se o quanto é limitado o pensamento linear e causal.

Da mesma forma pode-se pensar o processo saúde-doença. Franco e Bastos (2002), ao apresentarem uma proposta de articulação da abordagem ecológica de desenvolvimento com o modelo de vigilância da saúde, apontam que a saúde da pessoa encontra-se numa equação com a saúde do grupo e da comunidade na qual esta se insere e consideram o indivíduo como ativo no 
processo saúde-doença que, por sua vez, está vinculado a uma gama extensa de determinantes socioambientais.

Assim, ao refletir sobre os fatores determinantes do desenvolvimento saudável de uma criança, além das condições biológicas inatas, é preciso pensar sobre a influência familiar, da comunidade na qual está inserida e aspectos mais amplos da conjuntura socioeconômica e política sem perder de vista as transformações temporais e históricas de cada um desses contextos.

\section{Fatores de risco para 0 desenvolvimento}

Nas últimas décadas tem-se assistido a uma preocupação dos pesquisadores com o desenvolvimento infantil e com os diversos fatores negativos aos quais as crianças estão expostas. Conforme apontam Halpern, Giugliani, Victoria, Barros e Horta (2000), para as crianças que vivem em países em desenvolvimento, esses fatores incluem riscos como o de terem alta prevalência de doenças, de nascerem de gestações desfavoráveis e o de viverem em situações econômicas adversas, tendo maior chance de apresentar problemas no crescimento e desenvolvimento. Zamberlam e Biasoli-Alves (1996) corroboram com essa idéia afirmando que condições de pobreza, subnutrição, habitação inadequada, rupturas familiares, ausência de educação, abuso e violência são fatores de risco aos quais estão expostas muitas crianças brasileiras.

Segundo Grizendko e Fisher (1992, citados por Halpern \& Figueiras, 2004), fator de risco é um elemento que, quando presente, aumenta a probabilidade de surgimento de problemas ou aumenta a vulnerabilidade de uma pessoa ou grupo a desenvolver uma doença ou agravo à saúde.

Hanson e Lynch (1989, citados por Graminha \& Martins, 1997) consideram três tipos de condição de risco para o atraso no desenvolvimento:

- Risco Biológico: referindo-se a eventos pré, peri e pós-natais;

- Risco Estabelecido: desordens médicas especialmente de origem genética;

- Risco Ambiental: condições precárias de saúde, poucos recursos sociais.
De acordo com Sameroff e Seifer (1983), a visão inicial sobre risco estava focada nas condições médicas da criança e o desvio no desenvolvimento era considerado uma característica da criança independente do contexto; a noção de risco recente considera a interação dos fatores da criança e do contexto ambiental, tratando-se de uma transação entre o individual e o ambiental mais do que algo do indivíduo em si. Esses autores destacam fortemente a importância de se considerar a influência do contexto ambiental e sua forma de organização sobre o desenvolvimento da criança.

Segundo Lewis, Dlugokinski, Caputo e Griffin (1988), para que se tenha um modelo de compreensão e intervenção a respeito dos fatores de risco e de proteção, devem ser consideradas as seguintes dimensões: a) fatores de base da criança (que incluem os componentes biológicos, temperamento, sintomatologia e desenvolvimento de competência); b) fatores da família nuclear (que incluem história adaptativa dos pais, estressores e coesão familiar) e c) fatores do ambiente mais amplo (como suporte social da família ampliada, vizinhos e ambiente escolar).

Para Garbarino (1990, citado por Halpern \& Figueiras, 2004) falar sobre fatores de risco implica em considerar dois tipos de interação: primeiramente, num sentido mais restrito, a interação no microssistema, da criança com a família. Posteriormente, num sentido mais amplo, relacionamento do microssistema com os demais (exossistema, macrossistema e cronossistema). Esses tipos de interação demonstram as várias influências e a complexidade envolvida no desenvolvimento infantil e em seus riscos de prejuízo.

A partir dessa compreensão fica fácil perceber como os resultados negativos no desenvolvimento são produzidos muitas vezes não só por um, mas pela combinação de mais de um fator de risco. Os diferentes fatores que determinam os problemas que a criança apresenta ao longo de seu desenvolvimento são mais dependentes da quantidade do que da natureza dos fatores de risco, reforçando a importância de constatarmos os riscos múltiplos, que por sua vez têm efeito cumulativo causando um impacto maior sobre o desenvolvimento.

Laucht, Esser e Schmidt (2001) acompanharam longitudinalmente o comportamento de crianças nascidas com risco biológico (baixo peso ao nascer) e risco psicossocial (desvantagem fami- 
liar). A intenção era examinar o papel da responsividade dos cuidados iniciais na busca de fatores que moderem os efeitos de risco inicial. A responsividade materna foi apontada como moderadora do efeito de baixo peso, reduzindo a hiperatividade e os problemas de internalização, reforçando a importância de se preservar o vinculo mãe-bebê. Os resultados oferecem evidências de que os fatores familiares adversos têm forte impacto negativo sobre o desenvolvimento da criança. Além disso, os pesquisadores sugerem, a partir dos resultados, que quando há riscos psicossociais e biológicos conjugados, este efeito simultâneo é amplamente aditivo, afetando o desenvolvimento da criança até a idade escolar.

As pesquisas nacionais de autores como Zamberlam e Biasoli-Alves (1996), Graminha e Martins (1997), Puccini, Wecher e Silva (1997), Halpern et al. (2000), Miranda et al. (2003), Halpern e Figueiras (2004), Martins, Costa, Saforcada e Cunha (2004), dentre outros, têm contribuído para a identificação dos fatores de risco aos quais estão expostas as crianças brasileiras, mostrando a importância de avaliar a exposição das crianças a vários fatores de risco associados e o impacto do efeito cumulativo dos riscos. Esses estudos também chamam atenção para a importância do diagnóstico precoce, da prevenção e do tratamento e acompanhamento adequado para as crianças em situação de risco.

Embora os trabalhos de identificação dos fatores de risco para o desenvolvimento sejam fundamentais, é preciso direcionar o foco das pesquisas também para os fatores que podem proteger o desenvolvimento, fornecendo subsídios importantes para o planejamento de ações preventivas na atenção básica em saúde.

No "modelo transacional" de desenvolvimento, proposto por Sameroff e Chandler (1975), citados por Sameroff e Seifer (1983) e Halpern et al. (2000), a criança e o ambiente são vistos como ativamente engajados um com outro, mudando e sendo mudados por suas interações e o desenvolvimento é visto como único e peculiar, tendo como resultado o balanço entre os fatores de risco e os de proteção, da criança e do contexto.

O conceito de fatores de proteção aparece no trabalho de Rutter (1987), relacionado com a noção de "resiliência". Segundo o autor, o termo resiliência descreve o pólo positivo das diferenças individuais nas respostas ao estresse e adversida- de. A resiliência, ou capacidade de recuperação, ocorre em parte devido aos processos de proteção e vulnerabilidade pelos quais há modificação catalisadora de uma resposta do indivíduo à situação de risco.

Rutter (1987) destaca a mudança de foco do conceito de vulnerabilidade para o de resiliência como reflexo da inserção de esperança e otimismo no campo da adversidade, quando a atenção é voltada para os mecanismos de proteção. O autor chama atenção para o uso dos termos "mecanismos" e "processos" de proteção no lugar de "fatores" ou "variáveis", enfatizando a existência de mecanismos situacionais nos processos de proteção. Da mesma forma, enfatiza que resiliência não é um atributo fixo de um indivíduo, mas está relacionado com as circunstâncias existentes.

Os conceitos de vulnerabilidade e mecanismos de proteção estão relacionados a mudanças na resposta da pessoa à situação de risco e são os pólos negativo e positivo do mesmo conceito. Enquanto vulnerabilidade intensifica a reação à situação de risco, o mecanismo de proteção ameniza esta reação. O efeito de ambas é indireto e dependente de algum tipo de interação, sendo evidente somente em combinação com o fator de risco (Rutter, 1987).

Segundo Rutter (1987), os processos de proteção incluem: 1) os que reduzem o impacto de uma cadeia negativa de relações que envolvem o risco a) alterando o significado ou perigo do risco para a criança ou b) alterando a exposição ou envolvimento com a situação de risco; 2) os que reduzem a cadeia negativa de reações ao risco; 3) os que promovem e mantêm auto-estima e auto-eficácia por meio da disponibilidade de relacionamentos pessoais de apoio e segurança e 4) os que abrem oportunidades.

Segundo Zamberlam e Biasoli-Alves (1996), os fatores relativos à afetividade, harmonia conjugal e suporte sociofamiliar seriam caracterizados como fatores protetores às condições de risco e sua presença e intensidade influenciariam uma condição de responsividade diferencial de cada sujeito à situação.

Garmezy (1985), citado por Rutter (1987), faz uma revisão de pesquisas e lista três tipos de variáveis que operam como fatores de proteção: 1) características de personalidade, como auto-estima; 2) coesão familiar e ausência de discórdia e 3) disponibilidade de sistemas de apoio externos. 
Os fatores de proteção residem na maneira pela qual o indivíduo lida com as mudanças de vida e como age diante de circunstâncias de desvantagem e estresse (Rutter, 1987). Assim, uma atenção particular precisa ser dispensada aos mecanismos que operam nos pontos importantes de mudança na vida das pessoas, redimensionando as trajetórias de risco, como as ações em atenção básica, vigilância do desenvolvimento e promoção à saúde.

\section{Ações em Atenção Bá sica - Vigilância do Desenvolvimento e Promoção à Saúde}

Após a apresentação das reflexões anteriores acerca do desenvolvimento infantil, percebemos claramente o quão complexos e amplos são os fatores determinantes da saúde integral da criança. A partir daí pode-se melhor apreender a difícil tarefa dos sistemas de saúde diante dessa realidade multideterminada.

As ações estratégicas do Ministério da Saúde na atenção a criança vêm se ampliando gradativamente a partir da organização da atenção básica. A partir de 1984, foram priorizadas cinco ações básicas de saúde infantil (promoção do aleitamento materno, acompanhamento do crescimento e desenvolvimento, imunizações, prevenção e controle das doenças diarréicas e das infecções respiratórias agudas), como o centro da atenção a ser prestada em toda a rede básica de serviços de saúde. A partir de 1996, com a promoção da organização da atenção básica nos municípios junto ao PSF, as normas para o acompanhamento do crescimento e desenvolvimento foram gradativamente incorporadas às atividades dos profissionais da atenção básica, potencializando os esforços para a vigilância da saúde da criança, com destaque para a disseminação do uso do Cartão da Criança (Brasil. Ministério da Saúde, 2002).

As ações de atenção básica devem estar pautadas em um entendimento complexo do desenvolvimento infantil e voltadas para a promoção à saúde e prevenção. Segundo Figueiras, Puccini, Silva e Pedromônico (2003), no caso de problemas do desenvolvimento, para que haja intervenção precoce é necessário identificar as crianças e encaminhá-las o mais breve possível para serviços especializados. "Portanto, é papel do pro- fissional que atua na atenção primária, fazer a vigilância do desenvolvimento de todas as crianças, identificar aquelas com necessidades especiais e encaminhá-las oportunamente para tratamento" (Figueiras et al., 2003, p. 1692).

O Ministério da Saúde, numa proposta de acompanhamento do crescimento e desenvolvimento da criança, coloca que

Cada contato entre a criança e os serviços de saúde, independente do fato, queixa ou doença que o motivou, deve ser tratado como uma oportunidade para a análise integrada e preditiva de sua saúde, e para uma ação resolutiva, de promoção da saúde, com forte caráter educativo (Brasil. Ministério da Saúde, 2002, p. 27).

Na publicação "Agenda de Compromissos para a Saúde Integral da Criança e Redução da Mortalidade Infantil", o Ministério da Saúde (2004) contempla as diversas necessidades que discutimos neste trabalho, colocando a criança como foco de atenção em diversas oportunidades, seja na unidade de saúde, no domicílio ou nos espaços coletivos, beneficiando-se de um cuidado integral e multiprofissional. Como princípios norteadores aparecem: o desenvolvimento de ações intersetoriais; o acesso universal; o acolhimento; a assistência integral e resolutiva; atuação em equipe; participação da família, entre outros. As linhas de cuidado incluem desde a atenção à gestante e ao recém-nascido e incentivo ao aleitamento materno até incentivo e qualificação do acompanhamento do crescimento e desenvolvimento $(\mathrm{CD})$ e atenção à saúde mental. Como estratégias de ação são mencionadas, dentre outras, a vigilância à saúde pela equipe de atenção básica e a educação continuada das equipes de atenção primária.

Numa pesquisa realizada em Belém foram avaliadas as práticas e conhecimentos de profissionais de atenção primária à saúde, sobre vigilância do desenvolvimento infantil e concluiu-se que os médicos e enfermeiras participantes (160 profissionais) apresentaram deficiências nos conhecimentos sobre desenvolvimento infantil e que a vigilância do desenvolvimento não é realizada de forma satisfatória (Figueiras et al., 2003).

Halpern e Figueiras (2004), em seu artigo de revisão sobre as influências ambientais na saúde mental da criança, também afirmam que a maioria dos profissionais de atenção primária não avalia 
o desenvolvimento e a saúde mental da criança na sua prática diária, apontando para a importância dos programas de intervenção precoce abrangendo crianças nos principais grupos de risco: a) grupos com diagnósticos estabelecidos, como as síndromes diversas; b) grupo com risco biológico relacionado às condições de gestação e nascimento e c) grupo com riscos sociais relacionados com condições socioeconômicas desfavoráveis, estrutura familiar deficiente e cuidado inadequados.

Segundo Figueiras et al. (2003), a operacionalização da vigilância do desenvolvimento seria facilitada se os serviços de saúde normatizassem algumas condutas, principalmente para crianças de até dois anos, buscando identificar precocemente qualquer problema.

Mesmo sendo necessário reconhecer as dificuldades que permeiam as intervenções precoces em países em desenvolvimento, existem inúmeras alternativas para promover programas de baixo custo e de abordagem comunitária envolvendo as crianças em situação de risco (Halpern \& Figueiras, 2004).

Franco e Bastos (2002) em seu trabalho da abordagem ecológica do desenvolvimento com o modelo de vigilância em saúde buscam elaborar uma ferramenta para o Programa de Saúde da Família - PSF. O modelo de vigilância da saúde visa a organizar as ações de saúde priorizando a ação sobre os fatores de risco. Tal modelo enfatiza o papel do ambiente e pressupõe um modo de lidar com a saúde sobre os princípios da prevenção de danos, procurando enfatizar e ampliar os aspectos saudáveis.

A respeito dos fatores de risco para o desenvolvimento, o Ministério da Saúde também os toma como importantes, como na seguinte afirmação:

A identificação de um ou mais fatores de risco, tais como: baixo peso ao nascer, baixa escolaridade materna, idades maternas extremas $(<19$ anos e $>35$ anos), gemelaridade, intervalo intergestacional curto (inferior a dois anos), criança indesejada, desmame precoce, mortalidade em crianças menores de 5 anos na família, condições inadequadas de moradia, baixa renda e desestruturação familiar exigem um acompanhamento especial, pois aumentam a probabilidade da existência de doença perinatal e infantil (Brasil. Ministério da Saúde, 2002, p. 27).
O setor de saúde deve, em trabalho conjunto com outros setores organizados e com participação da população local, identificar e atuar sobre os riscos aos quais a população está submetida e com os grupos de riscos para danos específicos à saúde. Deve haver uma redefinição do objeto do modelo assistencial. Na saúde coletiva, tratase de investir em ações e práticas de saúde com foco no coletivo, no cotidiano da população, considerando seus membros como cidadãos e sujeitos ativos e sociais (Franco \& Bastos, 2002).

Nesse sentido Halpern e Figueiras (2004, p. 107) também fazem a seguinte afirmação:

A intervenção sobre os fatores de risco e mecanismos de ação que influenciam a saúde mental da criança extrapola a área de saúde. Cada vez mais se confirma a necessidade de se atuar intersetorialmente, envolvendo as áreas de saúde, educação, assistência social e econômica, com geração de emprego e renda para as famílias, de infra-estrutura ambiental e de lazer. Projetos integrados com objetivos de promover o bom desenvolvimento das crianças e adolescentes têm sido implementados em diversas comunidades, principalmente nas de menor nível socioeconômico, demonstrando uma alta probabilidade de êxito.

Baseado no atual modelo de organização do sistema de saúde pública no Brasil, Souza e Carvalho (2003) procuraram registrar os impactos das ações do PFS na saúde da população de Minas Gerais. Mediante uma observação participante as autoras concluíram que as intervenções do PSF procuram se basear nas características particulares das pessoas atendidas. O exercício da rotina inclui a prática do inquérito e das visitas domiciliares, o qual oportuniza conhecer a realidade das famílias. A aproximação das equipes de saúde com a comunidade é muito importante quando se percebe que quanto mais a intervenção for congruente com a demanda melhores podem ser os resultados. As autoras preconizam que a contextualização das ações é um elemento inscrito no trabalho do PSF por meio de uma postura de observação, sensibilidade e flexibilidade dos profissionais envolvidos. O caráter contextual das ações é essencial na busca de incentivos à participação e ao controle social, fatores também que corroboram com o novo modelo de atenção. Ao se identificar com a proposta, a população se coloca mais disposta a par- 
ticipar e a apoiar as ações que dizem respeito a problemáticas pessoais, o que favorece a mobilização e o engajamento de todos.

Articulando as idéias apresentadas, cabe novamente ressaltar a importância da família que, por ser o sistema e contexto básico de desenvolvimento, deve ser foco dos programas de intervenção e principalmente de promoção à saúde. Quando bem orientada e com o devido suporte, a família pode ser promotora do desenvolvimento saudável da criança, pois os cuidados familiares podem evitar os riscos e servir como fatores de proteção para o desenvolvimento.

Franco e Bastos (2002) apontam a família como unidade de informação e ação, unidade de cuidado do PSF e primeiro sistema constituidor do desenvolvimento humano. Assim a família é objeto de cuidados, mas sua condição é ativa. Ela é constituidora de si e de seus membros, cuidando e preservando sua sobrevivência.

Falar da família como agente de seus próprios cuidados abre uma nova discussão, com a exigência de repensar o lugar do serviço de saúde, do profissional, do controle social e da família, e assim coletivamente "preparar canções para acordar os homens e adormecer as crianças" (Carlos Drummond de Andrade) (Franco \& Bastos, 2002, p. 71).

Halpern e Figueiras (2004) mencionam a importância do papel dos médicos clínicos e dos pediatras da identificação e intervenção dos problemas de saúde e desenvolvimento da criança, devido a sua possibilidade de acompanhamento contínuo, apontando para a importância do envolvimento desses profissionais na promoção à saúde mental das crianças. A partir dessa contribuição, podemos pensar de modo mais amplo na importância do envolvimento do médico de família e de todos os profissionais de uma equipe de PSF. Esses profissionais sim estão em uma posição privilegiada para fazer um acompanhamento integral da criança, pois além de acompanhá-la continuamente, acompanham juntamente toda a família e a comunidade imediata, conhecendo de perto os sistemas influentes em seu desenvolvimento e, além disso, têm a possibilidade de ação interdisciplinar.

A partir dos dados discutidos fica clara a necessidade dos serviços de saúde voltarem sua atenção à promoção da saúde e desenvolvimento da criança de forma mais eficaz. É importante que se vá além das estratégias preconizadas e que se parta para uma real efetivação de sistemas de vigilância do desenvolvimento, com capacitação dos profissionais, inclusão de outras categorias profissionais nos trabalhos de prevenção e promoção à saúde e envolvimento das famílias, comunidades e outros setores da sociedade além dos voltados exclusivamente à saúde.

\section{Considerações Finais}

Ao tentarmos, com esse texto, levantar reflexões importantes sobre o desenvolvimento infantil e sobre a forma como os sistemas de saúde precisam comprometer-se com a sua promoção, percebemos o quanto nosso país ainda precisa caminhar no sentido de desenvolver ações integrais de promoção à saúde da criança, assim como em outras áreas.

Por outro lado, o modelo do PSF, ao contar com uma equipe multiprofissional e com a possibilidade de trabalho interdisciplinar, cria importantes ferramentas para realizar adequadamente ações de promoção à saúde da criança além da vigilância do desenvolvimento, aproveitando sua inserção na comunidade e podendo constituir-se efetivamente numa equipe que trabalha "ecossistemicamente".

É preciso pensar ainda que, para que essas ações possam ser implementadas de forma efetiva, são necessárias contribuições de trabalhos de pesquisa nessa área, pois só através do conhecimento da realidade é que se pode agir. Nesse sentido, para que essas contribuições das pesquisas possam otimizar ainda mais as ações em atenção básica - vigilância do desenvolvimento e promoção à saúde, seria muito útil a adoção do paradigma sistêmico, um pensamento que contemple a complexidade e contextualização; a instabilidade e a indeterminação; a interjubjetividade e a multiversa, ou seja, as diferentes versões possíveis para o fenômenos que envolvem a saúde e a doença.

Cabe enfatizar ainda a importância do conhecimento psicológico e atuação do psicólogo nas ações voltadas para a promoção da saúde da criança. A psicologia pode contribuir com as equipes de saúde ajudando na compreensão da importância do vínculo família-criança e, mais amplamente, do vínculo família-profissional de saú- 
de. A partir dessa compreensão, pode-se mais facilmente implementar ações de prevenção e promoção à saúde, com avaliação e estimulação do desenvolvimento como ações que, a partir de orientações adequadas, podem ser realizadas por todas as pessoas que cuidam da criança, incluindo familiares, profissionais de saúde, educadores, entre outros, em todos os contextos em que a criança convive e em diversos setores da comunidade.

A partir do seu conhecimento sobre o desenvolvimento infantil e sobre as interações familiares, o profissional de psicologia pode integrar equipes de atenção básica e contribuir amplamente nos trabalhos de prevenção de problemas do desenvolvimento de forma integral, incluindo o desenvolvimento psicológico e afetivo da criança e da família. Ao estar atento às interações familiares que promovem a saúde e o desenvolvimento da criança ou protegem contra riscos, o psicólogo pode ajudar a equipe de saúde a amplificá-las, além de intervir sobre as interações nos diversos contextos que expõem as crianças à vulnerabilidade.

\section{Referências}

Brasil. Ministério da Saúde. (1997). Saúde da Família: Uma estratégia para reorientação do modelo assistencial. Brasília: Ministério da Saúde.

Brasil. Ministério da Saúde. Secretaria de Políticas de Saúde. Departamento de Atenção Básica. (2002). Saúde da criança: Acompanhamento do crescimento e desenvolvimento infantil. Brasília: Ministério da Saúde.

Brasil. Ministério da Saúde. Secretaria de Atenção à Saúde. Departamento de Ações Programáticas Estratégicas. (2004). Agenda de compromissos para a saúde integral da criança e redução da mortalidade infantil. Brasília: Ministério da Saúde.

Bronfenbrenner, U. (1994). Ecological models of human development. In: International Encyclopedia of Education. (Vol. 3, 2nd ed., pp.1643-1647). Elsevier Sciences, Oxford, England.

Bronfenbrenner, U. (1996). A ecologia do desenvolvimento humano: Experimentos naturais planejados. Porto Alegre: Artes Médicas.
Figueiras, A. C. M., Puccini, R. F., Silva, E. M. K., \& Pedromônico, M. R. M. (2003). Avaliação das práticas e conhecimentos sobre vigilância do desenvolvimento infantil. Cadernos de Saúde Pública, 19(6), p.1691-1699.

Franco, A. L. S., \& Bastos, A. C. S. (2002). Um olhar sobre o programa de saúde da família: a perspectiva ecológica na psicologia do desenvolvimento segundo Bronfenbrenner e o modelo da vigilância da saúde. Psicologia em Estudo $7(2), 65-72$.

Graminha, S.S.V., \& Martins, M.A.O. (1997). Condições adversas na vida de crianças com atraso no desenvolvimento. Medicina, Ribeirão Preto, 30(2), 259-267.

Halpern, R., \& Figueiras, A. C. M. (2004). Influências ambientais na saúde mental da criança. Jornal de Pediatria 80,2 (Supl), S104-S110.

Halpern, R., Giugliani, E., Victora, C. G., Barros, F., \& Horta, B. (2000). Fatores de risco para suspeita de atraso no desenvolvimento neuropsicomotor aos 12 meses de vida. Jornal de Pediatria, 76(6), 421-428.

Laucht, M., Esser, G., \& Schmidt, M. (2001). Differential development of infants at risk for psychopathology: the moderating role of early maternal responsivity. Development Med Child Neurology 43(5), 292-300.

Lewis, R.J.; Dlugokinski, E.L.; Caputo, L.M.; \& Griffin, R. B. (1988). Children at risk for emotional disorders: risk and resource dimensions. Clinical Psychology Journal 8, 417-440.

Martins, M.F.D., Costa, J.S.D., Saforcada, E.T., \& Cunha, M. D. C. (2004). Qualidade do ambiente e fatores associados: um estudo em crianças de Pelotas, Rio Grande do Sul, Brasil. Cadernos de Saúde Pública, 20(3), 710-718.

Miranda, L. P., Resegue, R., \& Figueiras, A. C. M. (2003). A criança e o adolescente no ambulatório de pediatria. Jornal de Pediatria 79 (Supl.1), S33-S42.

Puccini, R. F., Wecher, R., Silva, E. M.K., \& Resegue, R. (1997). Fatores de risco para morbidade e desnutrição em crianças acompanhadas em programas de atenção à saúde da criança. Jornal de Pediatria 73 (4), 244-251. 
Rutter, M. (1987). Psychosocial resilience and protective mechanisms. American Journal Orthopsychiatry, 57(3), 316-331.

Sameroff, A., \& Seifer, R. (1983). Familial Risk and Child Competence. Child Development, 54(5), 1254-1268.
Souza, R. A., \& Carvalho, A. M. (2003). Programa de Saúde da Família e qualidade de vida: um olhar da psicologia. Estudos de Psicologia, 8(3), 515523.

Zamberlan, M. A. T., \& Biasoli-Alves, Z. M. (1996). Interações familiares: Teoria, pesquisa e subsídios à intervenção. Londrina: Ed. UEL.

Recebido em/ received in: 01/07/2005

Aprovado em/ approved in: 02/08/2005 